\title{
A red swollen forearm in an elderly man
}

A 90-year-old man with chronic kidney disease, ischaemic heart disease, hypertension and a history of recurrent falls presented with a circumferential, erythematous and indurated right forearm plaque for 2 weeks (Fig. 1). Pinprick sensation was intact over the lesion and the rest of the upper limbs. His left arm was not affected. He fell 3 months prior to this episode and sustained 2 right forearm lacerations, which had healed with scarring before he noticed the plaque. It did not improve after a week's course of oral amoxicillinclavulanic acid given by his primary care provider. $\mathrm{He}$ was afebrile and physical examination was otherwise unremarkable. Laboratory investigations revealed a white cell count of $6.9 \times 10^{3} / \mathrm{uL}$ with no neutrophilia or eosinophilia, c-reactive protein of $1.9 \mathrm{mg} / \mathrm{L}$, and procalcitonin of $0.08 \mu \mathrm{g} / \mathrm{L}$. A Doppler venous ultrasound and right forearm X-ray were performed. These were reported as normal. A skin biopsy was performed for histologic examination (Figs. 2 and 3) as well as tissue cultures.

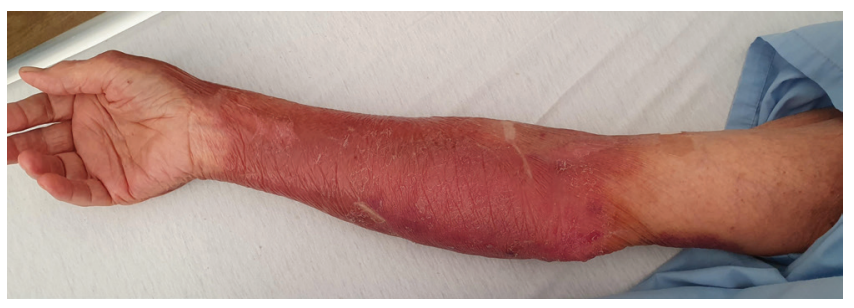

Fig. 1. Circumferential erythema and oedema arising from a background of linear scars and senile purpura on the right forearm.

What is the most likely diagnosis?
A. Borderline leprosy
B. Angiosarcoma
C. Eosinophilic cellulitis (Wells syndrome)
D. Extensive senile purpura
E. Non-tuberculous mycobacterium infection

We suspected an infection with an atypical organism because of the history of recurrent skin trauma, degree of induration and non-response to amoxicillinclavulanic acid.

Skin biopsy revealed a necrotising granulomatous inflammation in the dermis (Fig. 2) with a few acid-fast bacilli demonstrated on Fite stain (Fig. 3). A rapidly growing non-tuberculous mycobacterium was isolated from the bacterial, fungal and mycobacterial skin tissue cultures. This was identified as Mycobacterium fortuitum. The isolate was sensitive to imipenem, amikacin, ciprofloxacin, moxifloxacin, trimethoprim/ sulfamethoxazole and doxycycline. It was resistant to clarithromycin, likely from an inducible ribosomal RNA methylase $(\mathrm{erm})$ gene. ${ }^{1}$ The patient received empirical oral clarithromycin initially but developed bicytopaenia, which was attributed clinically to clarithromycin. He was switched to oral doxycycline and ciprofloxacin, but developed non-specific symptoms, which he attributed to doxycycline. He eventually received oral ciprofloxacin $500 \mathrm{mg}$ daily, with a plan to complete 6 months. The redness and swelling on his right arm were almost resolved when we reviewed him 3 months after treatment.

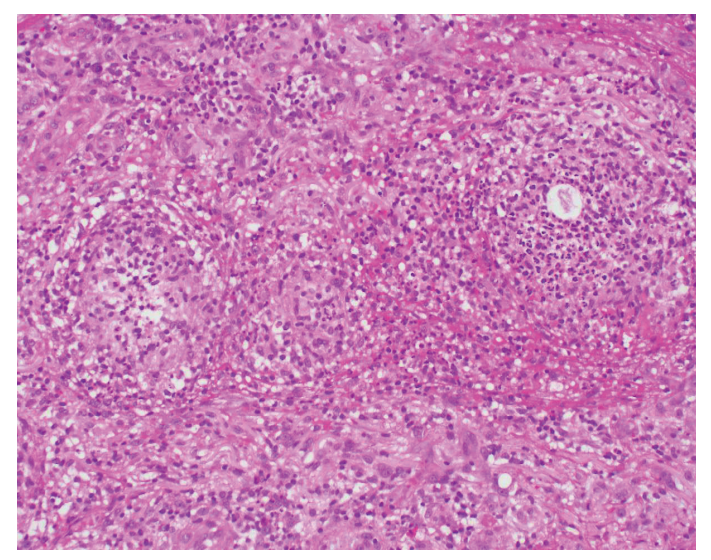

Fig. 2. Histological examination revealed a necrotising granulomatous infiltrate in the dermis (haematoxylin and eosin stain, magnification 400X).

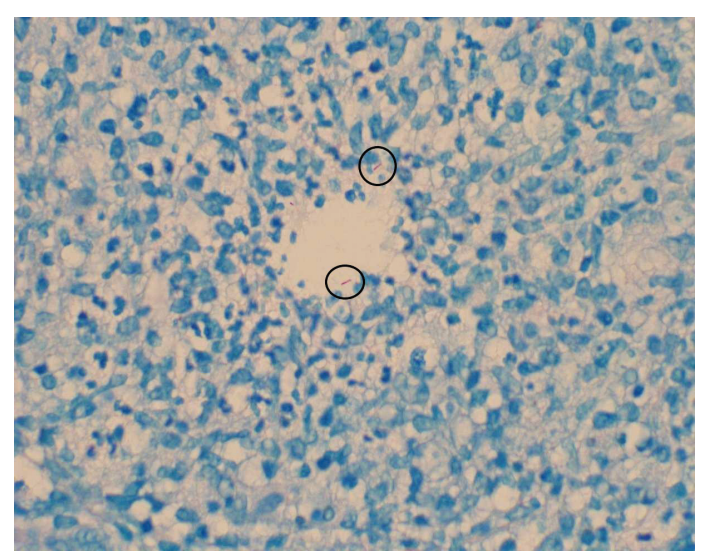

Fig. 3. A few acid-fast bacilli (circled) on Fite stain (magnification 600X). 
M. fortuitum accounted for $19 \%$ of non-tuberculous mycobacterial skin infection cases reviewed in a Singapore study. ${ }^{2}$ This rapid-growing mycobacterium is a saprophytic organism that causes infection following traumatic inoculation from contaminated surfaces or instruments. Cutaneous infection presents as nodules, abscesses, ulcers or cellulitis. A high index of suspicion for infection with uncommon organisms such as $M$. fortuitum is required when confronted with treatment-resistant cellulitis, especially if it was preceded by a recent skin injury. Diagnosis is confirmed with histological examination and mycobacterial culture of affected tissue, as was done for this case. The recommended treatment regime for $M$. fortuitum skin infection is at least 4 months of treatment with at least 2 antibiotics guided by the antimicrobial sensitivity analysis of the clinical isolate, in combination with surgical removal of any retained foreign bodies or drainage of abscesses. ${ }^{3}$

The clinical presentation of leprosy falls within a spectrum that is determined by the interaction between the organism and the host immune system. ${ }^{4}$ Borderline leprosy can present on the skin with several asymmetrically distributed annular erythematous plaques with associated reduced sensation and hair growth. The peripheral nerves may be thickened. Histological examination often reveals a granulomatous inflammatory infiltrate surrounding the cutaneous nerves with very few acid-fast bacilli in tuberculoid leprosy, or a generalised dermal lymphohistiocytic infiltrate sparing a narrow area of the papillary dermis (grenz zone) and numerous acid-fast bacilli in clumps (globi) within histiocytes in lepromatous leprosy. The points against this diagnosis in our patient are the acuity of the presentation, lack of sensory loss and lack of multiple lesions. The histology examination was not consistent with leprosy as well.

Angiosarcoma in the elderly often presents on the face and scalp as chronic erythematous plaques that progress to tumours or ulcers over time. ${ }^{5}$ Angiosarcoma involving the limbs occurs in the context of chronic lymphoedema (e.g. from prior ipsilateral mastectomy with axillary lymph node clearance) or after radiotherapy. Our patient presented acutely and did not have a history of preceding chronic lymphoedema or irradiation to the right arm. The histology examination was not consistent with angiosarcoma as well.

Wells syndrome or eosinophilic cellulitis presents with an acute itchy cellulitis-like plaque that remits and relapses. ${ }^{6}$ It does not respond to antibiotics and is associated with peripheral eosinophilia. Histology of the affected skin reveals an eosinophilic dermal infiltrate with flame figures consisting of eosinophilic major basic protein coating the dermal collagen. Although the acute nature of our patient's presentation and lack of response to antibiotics might suggest this diagnosis, the histological findings and tissue cultures led to the correct diagnosis of $M$. fortuitum infection.

Extensive purpura on areas of cutaneous trauma can be seen in elderly patients with recurrent falls, especially if there is concomitant use of anti-platelet or anticoagulation agents, or in the context of systemic diseases causing fragile dermal blood vessels (e.g. scurvy or systemic amyloidosis), thrombocytopenia (e.g. myelodysplastic syndrome or dengue), coagulopathy (e.g. chronic liver disease) or skin atrophy (e.g. after systemic or topical steroid use). Our patient had clinical swelling and induration of the affected skin which are not typically seen in senile purpura. This observation should alert the clinician to consider an alternative cause.

Our case highlights the differential diagnoses to consider for patients with an upper limb cellulitis that does not respond to empirical antibiotic therapy. Infections with atypical organisms should be considered and confirmed with tissue histology and cultures.

\section{REFERENCES}

1. Nash KA, Zhang Y, Brown-Elliott BA, et al. Molecular basis of intrinsic macrolide resistance in clinical isolates of Mycobacterium fortuitum. J Antimicrob Chemother 2005;55:170-7.

2. Yeo PM, Lee SX, Tan YE, et al. Epidemiology, risk factors, and outcomes of adult cutaneous non-tuberculous mycobacterial infection over a 10-year period in Singapore. Int J Dermatol 2019; 58:679-87.

3. Griffith DE, Aksamit T, Brown-Elliott BA, et al. An official ATS/IDSA statement: diagnosis, treatment, and prevention of nontuberculous mycobacterial diseases. Am J Respir Crit Care Med 2007; 175:367-416.

4. Maymone $\mathrm{MBC}$, Laughter $\mathrm{M}$, Venkatesh $\mathrm{S}$, et al. Leprosy: Clinical aspects and diagnostic techniques. J Am Acad Dermatol 2020;83:1-14.

5. Shustef E, Kazlouskaya V, Prieto VG, et al. Cutaneous angiosarcoma: a current update. J Clin Pathol 2017;70:917-25.

6. Moossavi M, Mehregan DR. Wells' syndrome: a clinical and histopathologic review of seven cases. Int J Dermatol 2003;42:62-7.

Wan Chen Ang $^{1}{ }_{M R C P}(U K)$,

Helen May Lin $\underline{\mathrm{Oh}}^{2}{ }_{F R C P(E d i n)}$, Chee Seng $\underline{\operatorname{Sim}}^{3}{ }_{F R C P A}$, Chia Chun $\underline{\text { Ang }}{ }^{1}{ }_{M R C P}(U K)$

\footnotetext{
'Department of Dermatology, Changi General Hospital, Singapore

${ }^{2}$ Department of Infectious Diseases, Changi General Hospital, Singapore

${ }^{3}$ Department of Laboratory Medicine, Changi General Hospital, Singapore
}

Correspondence: Dr Chia Chun Ang, Department of Dermatology, Changi General Hospital, 2 Simei Street 3, Singapore 529889.

Email: ang.chia.chun@singhealth.com.sg 